\title{
Multilokalität und gleichwertige Lebensverhältnisse: Handlungsansätze von Regionalplanung und -management
}

\section{Frank Othengrafen, Lena Greinke und Rainer Danielzyk}

Multilokalität ist als Phänomen nicht nur in urbanen, sondern auch in ländlichen Räumen vorzufinden (vgl. Lange 2018; Peer 2013). Das hängt v. a. mit vielfältigen Transformationsprozessen in ländlichen Räumen zusammen (Wiegandt/ Krajewski 2020; s.a. Beitrag Greinke/Lange/Born in diesem Band (Kap. 2)):

- Der wirtschaftsstrukturelle Wandel, d. h. der Bedeutungsverlust der Landwirtschaft, des dörflichen Handwerks, dorftypischer Dienstleistungen und lokaler Industrien führt oftmals zu einem Verlust von Arbeitsplätzen. Es kommt damit zur räumlichen Trennung von Wohnen und Arbeiten, was zu einem erhöhten Pendler*innenaufkommen, aber auch zur Entstehung multilokaler Lebensweisen beitragen kann (s.a. Danielzyk/Dittrich-Wesbuer 2020).

- Der demografische Wandel führt in vielen ländlichen Räumen zu einem Bevölkerungsrückgang und einer Veränderung der Bevölkerungszusammensetzung. In diesem Zusammenhang stellt sich vielerorts ganz konkret die Frage, wie die Versorgung mit Leistungen der technischen und sozialen Infra-

\section{F. Othengrafen $(\bowtie)$}

Technische Universität Dortmund, Fachgebiet Stadt- und Regionalplanung,

Dortmund, Deutschland

E-Mail: frank.othengrafen@tu-dortmund.de

L. Greinke

Institut für Umweltplanung, Leibniz Universität Hannover, Hannover, Deutschland E-Mail: greinke@umwelt.uni-hannover.de

R. Danielzyk

ARL - Akademie für Raumentwicklung in der Leibniz-Gemeinschaft, Hannover, Deutschland

E-Mail: danielzyk@arl-net.de 
strukturen zukünftig gesichert werden kann (u. a. BMVI 2015; Fachinger/ Künemund 2015).

- Der soziostrukturelle Wandel (z. B. zunehmender Anteil an Pendler*innenaufkommen, Individualisierung der Gesellschaft etc.) bedeutet u. a., dass familiäre und nachbarschaftliche Beziehungen an Bedeutung verlieren und insgesamt weniger Zeit für Engagement im Ort bleibt.

Diese Transformationsprozesse können zu unterschiedlichen räumlichen Auswirkungen führen, die durch mehrörtige Lebensweisen noch verstärkt werden (Danielzyk/Dittrich-Wesbuer 2020). So können multilokale Lebensweisen zur steigenden Inanspruchnahme von Wohn- und Siedlungsflächen durch Zweitwohnsitze beitragen sowie zunehmendes Verkehrsaufkommen verursachen. Zudem kann eine erhöhte Wohnraumnachfrage den Druck auf den lokalen oder regionalen Mietwohnungsmarkt erhöhen und gleichzeitig die Nachfrage in ländlichen Räumen stabilisieren. Darüber hinaus können phasenweise Abwesenheiten zur Fragmentierung von Dorfgemeinschaften und/oder zur Verarmung des ländlichen Soziallebens beitragen (vgl. Beitrag Greinke/Albrecht/Othengrafen/ Gutsche/Lehmann in diesem Band (Kap. 5)). Die skizzierten Entwicklungen gefährden dabei die raumplanerische (und gesellschaftlich gewollte) Zielsetzung, gleichwertige Lebensverhältnisse im Gesamtraum der Bundesrepublik Deutschland und in seinen Teilräumen zu gewährleisten. Dies wird zurzeit wieder intensiv mit Blick auf (vermeintlich) ,abgehängte Regionen“, insbesondere strukturschwache ländliche Räume, diskutiert (vgl. BMI 2019).

\subsection{Multilokalität und gleichwertige Lebensverhältnisse}

Der Abbau regionaler Disparitäten und die Schaffung gleichwertiger Lebensverhältnisse sind seit vielen Jahrzehnten eine zentrale Leitvorstellung der Raumordnung in der Bundesrepublik Deutschland. Darunter ,ist die Zielsetzung verstanden, die Lebensverhältnisse in allen Teilräumen einer größeren räumlichen Einheit mit staatlichen Maßnahmen so zu gestalten, dass die in jedem Teilraum erreichten Lebensverhältnisse von der dortigen Bevölkerung tendenziell als gleichwertig mit den Lebensverhältnissen in den anderen Teilräumen angesehen werden“ (Rosenfeld 2018: 838). Damit wird versucht, das sozialstaatliche Versprechen, jedefrau und jedermann am gesellschaftlichen Wohlstand teilhaben zu lassen, räumlich umzusetzen (Kersten 2006: 245). Allerdings zeigt sich hier 
bereits, dass sich (1) gleichwertige Lebensverhältnisse nicht automatisch einstellen, sondern politische Eingriffe räumliche Disparitäten aktiv fokussieren müssen (Rosenfeld 2018: 838) und dass (2) „eine vollständige Nivellierung der Lebensbedingungen weder möglich noch angestrebt [wird], weil regionale Vielfalt durchaus sinnvoll ist" (Scharmann et al. 2020: 3). Zudem gibt es (3) unterschiedliche Vorstellungen, welche Leistungen, Infrastrukturen und Dienstleistungen als zentral für die Realisierung von „Gleichwertigkeit“ anzusehen sind, was das Erreichen der Leitvorstellung gleichwertiger Lebensverhältnisse zusätzlich zu einer Herausforderung der Raumplanung macht.

Diese Diskussion wird bislang weitgehend losgelöst vom Phänomen Multilokalität geführt (Fischer 2020: 144). Dabei wird die Bedeutung des Phänomens für die räumliche Planung und die Sicherstellung gleichwertiger Lebensverhältnisse immer deutlicher (vgl. Beitrag Greinke/Albrecht/Othengrafen/ Gutsche/Lehmann in diesem Band (Kap. 5)), weil Multilokalität ,als raum-zeitlich strukturierter und strukturierender Rahmen der alltäglichen Lebensführung [...] auf elementare Lebens- und Planungsbereiche [wirkt]: Wohnen, Arbeit, Freizeit, Mobilität, soziale Beziehungen, Nachbarschaften, Quartiersentwicklung, Infrastrukturen, Raumentwicklung u. a.m.“ (Hilti 2009: 77). Die persönlichen, gesellschaftlichen und arbeitsmarktrelevanten Netzwerke erstrecken sich über immer größere (räumliche) Distanzen; verstärkt wird dieser Trend durch allgemein erhöhte Mobilitätsanforderungen und -möglichkeiten sowie zunehmende Digitalisierung in privatem und beruflichen Umfeld. Dies wiederum führt zu temporären An- und Abwesenheiten von Personen, die durch Tagespendeln, d. h. zirkuläre Alltagsmobilität, verursacht oder durch multilokale, also mehrörtige Lebensweisen hervorgerufen werden. Das Vorhandensein von sozialen und technischen Infrastrukturen hat dabei unmittelbare Auswirkungen auf Multilokalität: Beispielsweise können gut ausgebaute Infrastrukturen dazu beitragen, dass Multilokale komfortabel zwischen ihren Orten wechseln können (z. B. zwischen Metropolen und Großstädten). Gleichzeitig können fehlende Mobilitätsangebote v. a. in (funktional) peripheren Regionen die Reisemöglichkeiten, -geschwindigkeit und -ausgestaltung maßgeblich negativ beeinflussen, sodass Arbeitnehmer*innen evtl. nicht länger täglich pendeln können, sondern gezwungen sind, multilokal zu leben (s.a. Beitrag Othengrafen/Greinke/Lange/ Seitz in diesem Band (Kap. 1)). Damit stehen Kommunen vielerorts unmittelbar vor der Herausforderung, mit den Ursachen und Wirkungen multilokaler Lebensformen umzugehen. Ziel dieses Beitrages ist es deshalb, Multilokalität als potenzielles Handlungsfeld von Regionalplanung und -management zu identifizieren und - auch vor dem Hintergrund der Leitvorstellung gleichwertiger Lebensverhältnisse - zu diskutieren sowie den Umgang mit Multilokalität auf Regionsebene zu analysieren. 


\subsection{Potenzielle Handlungsfelder von Regionalplanung und -management}

Ein zentraler Akteur zur Sicherung gleichwertiger Lebensverhältnisse und der (öffentlichen) Daseinsvorsorge ist die Regionalplanung (vgl. ARL 2016), deren Aufgabe es ist, umsetzungsorientiert unterschiedliche Nutzungs- und Gestaltungsansprüche an Räume und Ressourcen durch integrierte Ansätze auszugleichen (Priebs 2018: 2047; Scharmann et al. 2020: 5). Sie bildet die formale Planungsebene zwischen Landesplanung und gemeindlicher Planung. Sie kann im Rahmen der Bereitstellung von Leistungen der Daseinsvorsorge durchaus auf Multilokalität und die damit verbundenen Auswirkungen eingehen und entsprechende planerische Festsetzungen treffen.

Dabei kommt v. a. dem Zentrale-Orte-Konzept als Instrument der Raumordnung eine zentrale Rolle zu (Blotevogel 2002; Terfrüchte/Flex 2018: 2975; Danielzyk/ Priebs 2020). Damit sollten und sollen für alle Bürger*innen in zumutbarer Entfernung Mindeststandards der Daseinsvorsorge gewährleistet werden (Rosenfeld 2018: 844; Scharmann et al. 2020: 4). Dazu zählen vor allem Einrichtungen des Bildungs- und des Gesundheitswesens sowie die Nahversorgung, aber auch z. B. Sport- und Kultureinrichtungen. Damit bietet das Zentrale-Orte-Konzept einen guten Ansatz, Voraussetzungen für ausgeglichene infrastrukturelle, soziale, wirtschaftliche, ökologische und kulturelle Verhältnisse zu schaffen ( 2 Abs. $2 \mathrm{Nr}$. 1 ROG). Insbesondere mit Blick auf (meist ländliche) Räume mit geringer Bevölkerungsdichte ist es folglich ein wichtiges raumordnerisches Konzept. Vielerorts führen prekäre Situationen mit unzureichender infrastruktureller Anbindung und Wegfall von Daseinsvorsorgeleistungen dazu, dass (junge) Menschen aus peripheren oder strukturschwachen Räumen abwandern oder multilokale Arrangements anstreben. Mit Blick auf die Infrastrukturausstattung kann das Konzept auch multilokale Lebensweisen „unterstützen“, denn eine gleichwertige Verteilung von Infrastrukturen kann dafür sorgen, dass Räume gut erreichbar sind und als Wohnstandorte fungieren können. Gleichzeitig stößt das Zentrale-Orte-Konzept immer öfter an seine Grenzen, v. a. wenn es darum geht, auf aktuelle Entwicklungen zeitgemäß und flexibel reagieren zu können (Blotevogel 2002) - dies umfasst u. a. auch hybride und fluide multilokale Lebensweisen, die sich nicht länger an überkommenen Siedlungsstrukturen und Mobilitätsformen orientieren und daher mit zentralörtlichen Kategorien nicht zu erfassen sind.

Aufgrund der immer komplexeren Aufgabenstellungen, Akteurskonstellationen und Prozessen ist seit über zwei Jahrzehnten offenkundig, dass das formelle Planungssystem zur Gestaltung der räumlichen Entwicklung längst nicht mehr aus- 
reicht (vgl. Priebs 2018: 2060f). Es wird daher ergänzt durch vielfältige Formen kooperativer, informeller Planungsansätze und -instrumente, die vor, parallel zu und nach formellen Planungen zum Einsatz kommen. Vor diesem Hintergrund existiert vielerorts ergänzend zur formellen Regionalplanung ein informelles Regionalmanagement, welches auch Handlungsansatz und Instrument der Raumordnung ist. Zudem wird ein Regionalmanagement ,,als [...] eine Institution zur Organisation kooperativer Prozesse in der Regionalentwicklung verstanden" (Heintel 2018: 2023). Ein Regionalmanagement verfolgt dabei das Ziel, die soziale, ökonomische, ökologische und raumstrukturelle Situation von Regionen in Richtung einer nachhaltigen Entwicklung zu beeinflussen. Dabei bringt ein Regionalmanagement initiativ und moderierend unterschiedliche Akteur*innen und Interessen - z. B. Städte, Gemeinden, Landkreise, Bürger*innen, Unternehmen, Umwelt-, Wirtschafts- und Sozialverbände - zusammen, um die Entwicklungspotenziale der Region durch Vernetzung besser zu nutzen (ebd.).

Herauszustellen sind in diesem Zusammenhang vor allem die Potenziale des Regionalmanagements in Bezug auf Multilokalität. Durch freiwillige Kooperationen und Vereinbarungen von öffentlicher Hand, insbesondere Kommunen, Wirtschaft und Zivilgesellschaft verändert sich das Verständnis regionaler Steuerung von hierarchischen hin zu „horizontalen“, kooperativen und ermöglichenden Steuerungsformen, die es erlauben, neue Handlungsoptionen für die Praxis zu entwickeln und zu etablieren (u. a. Blatter 2004; Einig et al. 2005; Fürst et al. 2006; Jann 2002). Dafür wird in den betroffenen Regionen über das Regionalmanagement oftmals ein Regionales Entwicklungskonzept (REK) erarbeitet, in dem eine Region beschrieben und analysiert sowie Entwicklungsperspektiven und -empfehlungen aufgezeigt werden (s.a. Scharmann et al. 2020: 5). Die REKs können folglich auch dazu genutzt werden 1.) die Anforderungen und Bedürfnisse multilokal lebender Personen in einen größeren Kontext einzubetten, 2.) Herausforderungen und Chancen mehrörtiger Lebensweisen zu erkennen und 3.) zusammen mit lokalen und regionalen Akteur*innen aus Planung, Politik und Verwaltung gezielte Handlungsansätze und -strategien zu entwickeln, um auf Multilokalität zu reagieren. Durch die Aufnahme der Thematik Multilokalität in die Konzepte kann zudem ein Beitrag zur Sensibilisierung aller beteiligten Akteur*innen für das Thema geleistet werden. Dies ist nötig, weil multilokale Lebensweisen in der derzeitigen Planungspraxis nur selten betrachtet werden und eine eher untergeordnete Rolle spielen. Bislang werden sie nur mit sehr wenigen Ausnahmen (z. B. Stadt Wolfsburg (vgl. Leubert 2020)) in kommunalen oder regionalen Konzepten überhaupt aufgenommen.

Kommunen, die über Multilokalisierungstreiber (z. B. Hochschulen, große Unternehmen mit mehreren überregional verteilten Standorten, große Behörden- 
standorte, Ferienhaussiedlungen, Campingplätze usw.) verfügen, haben oft zwar noch keinen Handlungsbedarf in Bezug auf Multilokalität identifiziert, sind aber zumeist schon in anderer Weise diesbezüglich aktiv, zum Beispiel in touristisch geprägten Gebieten. Beispielsweise haben sie bereits Zweitwohnsitzsteuern eingeführt oder auch Infrastrukturen für die Freizeitnutzung ausgebaut. Die Handlungsmöglichkeiten von Regionalplanung und -management bestehen hier vor allem in den Handlungsfeldern a) Bewusstseinsförderung und Ansprechstrukturen, b) Wohnen und Infrastrukturen, c) bürgerschaftliches Engagement sowie d) Arbeit und regionale Wirtschaft (vgl. Beitrag Greinke/ Albrecht/Othengrafen/Gutsche/Lehmann in diesem Band (Kap. 5) s.a. Oberösterreichische Zukunftsakademie 2019: 23), die im Rahmen von Regionalplanung und -management entsprechend berücksichtig oder adressiert werden können.

\section{Bewusstseinsförderung und Ansprechstrukturen}

Multilokale Lebensweisen sind derart komplex, dass es oft nicht ausreicht, nur vereinzelte Akteur*innen einzubinden. Aus diesem Grund ist es erforderlich, interkommunale Abstimmungsprozesse sowie darüber hinaus Austausch mit Unternehmen, Vereinen, Verbänden etc. anzustreben. Das Regionalmanagement kann hier dazu beitragen, verschiedene Akteur*innen zu vernetzen und zusammenzubringen. Mit Blick auf die Auswirkungen und Potenziale multilokaler Lebensformen steht v. a. die Betrachtung der Rahmenbedingungen im Vordergrund, die sowohl bürgerschaftliches als auch unternehmerisches Engagement für die lokale und regionale Entwicklung befördern sollten. Die Wirtschaftsförderung, hier verstanden als weitere Akteurin im Bereich der Regionalentwicklung, kann eine Kooperationspartnerin sein, mit der gemeinsam mögliche Handlungsansätze entwickelt und umgesetzt werden können. Die Regionalplanung hingegen ist nur bedingt handlungsfähig bzw. relevant, weil in den Kernaufgaben der Regionalplanung nicht unmittelbar immer multilokale Lebensweisen mitgedacht werden können. Beispielsweise sind aufgrund des Abstraktionsgrades der Regionalpläne nur wenig Aussagen hinsichtlich Multilokalität möglich. Das liegt zum einen daran, dass die Regionalplanung insbesondere überörtliche und überfachliche Aspekte betrachtet und dabei den Vorgaben der Landesplanung und des Zentralen-Orte-Konzeptes folgen muss. Multilokalität ist jedoch oft an individuelle Entscheidungen gekoppelt und lokal wirksam, aber nicht zwingend regional bedeutsam, sodass sie in der Angebotsplanung der Regionalplanung eine untergeordnete Rolle spielt. Zudem eignen sich formelle Regionalpläne nur bedingt zur Bewusstseinsbildung, weil sie nur die Fachöffentlichkeit ansprechen und nicht unbedingt als Kommunikationsstrategie oder -mittel konzipiert sind. 


\section{Wohnen und Infrastrukturen}

Um den Ansprüchen und Bedürfnissen mehrörtiger Lebensweisen gerecht $\mathrm{zu}$ werden, sind in Bezug auf das Handlungsfeld Wohnen und Infrastrukturen innovative Ansätze gefragt. Incomings, die phasenweise in einer Kommune leben, sind zumeist auf der Suche nach kleinen und gut erreichbaren Wohnungen als Zweitunterkunft, weil sie häufig berufsbedingt in die Kommunen einpendeln und dementsprechend einfache und flexible Wohnformen nachfragen. Wohnraumverfügbarkeit und Infrastrukturausstattung von Kommunen sind für Multilokale bedeutende Aspekte in ihrer Lebensweise und bei der Wahl der Orte (vgl. Beitrag Greinke/Lange in diesem Band (Kap. 4)). Dabei kann das Regionalmanagement Multilokalität mitdenken und im Rahmen eines Regionalen Entwicklungskonzeptes oder eines regionalen Wohnraumversorgungskonzepts (WRVK) den aktuellen Wohnungsmarkt und die zukünftigen Entwicklungen in den Kommunen analysieren (vgl. Beitrag Greinke/Lehmann/Othengrafen/Seitz in diesem Band (Kap. 7)). Insbesondere ressourcenschonende Wohnangebote sollten in den Fokus der Regionen rücken; dabei sollten Kommunen Stärkung der Ortszentren abzielen, um eine Zersiedlung zu vermeiden (vgl. Beiträge Greinke/Albrecht/Othengrafen/ Gutsche/Lehmann (Kap. 5) und Greinke/Lehmann/Othengrafen/Seitz in diesem Band (Kap. 7); s.a. Oberösterreichische Zukunftsakademie 2019: 30). Dazu bedarf es allerdings der politischen Unterstützung durch die Gemeinderäte. Die Regionalplanung ist in diesem Handlungsfeld nur bedingt handlungsfähig: Sie kann zwar über das Zentrale-Orte-System dafür sorgen, dass die Erreichbarkeit einer Region sichergestellt ist und dass Voraussetzungen für die räumliche Entwicklung von Regionen geschaffen werden. Sie trifft in den Regionalplänen aber lediglich allgemeine Festsetzungen (z. B. Grundzentrum mit mittelzentraler Teilfunktion für Wohnen und/oder Arbeitsstätten), die zwar Bindungswirkung haben, jedoch in ihrer Ausgestaltung nicht detailscharf sind. Zudem kann sie das Wachstum der Siedlungsflächen an den Orträndern begrenzen bzw. steuern. Die detailscharfe Gestaltung geschieht aber erst in den Kommunen durch die Ausübung der kommunalen Planungshoheit.

Da jedoch Wohnstandortanforderungen sehr unterschiedlich sind und zumeist nicht an einem Ort abgedeckt werden können, gilt es regionale Kooperationen auf- bzw. auszubauen. Dabei können Unterstützungsleistungen für Multilokale gemeinsam entwickelt werden, zum Beispiel regionale Wohnungsbörsen (vgl. Beitrag Greinke/Lehmann/Othengrafen/Seitz in diesem Band (Kap. 7)). In Bezug auf die regionale Infrastrukturausstattung gilt es für eine positive Regionalentwicklung, eine gute physische wie telekommunikative Erreichbarkeit (z. B. Fernverkehrsanschlüsse, ausreichende Breitbandverfügbarkeit) $\mathrm{zu}$ gewährleisten. Insbesondere Multilokale benötigen aufgrund ihrer mehrörtigen Lebensweise 
eine gut ausgebaute Infrastruktur für die An- und Abreise. Außerdem sind sie aufgrund der Entfernungen zwischen den Orten zumeist auf stabile Netze der Telekommunikation angewiesen, um kommunizieren zu können. Die Rolle von Regionalplanung und -management ist es hier, die Ansprüche und Bedürfnisse der lokalen Bewohner*innen und der Multilokalen in die zukünftigen Planungen einzubeziehen und abzuwägen.

\section{Bürgerschaftliches Engagement}

Multilokale können als Schlüsselakteur*innen gesehen werden, die die Lebensqualität der Orte positiv beeinflussen, sodass Abwanderungen gestoppt und ländliche Regionen in ihrer Entwicklung nachhaltig unterstützt werden können. Dabei übernehmen Multilokale, u.a. Outgoings, Verantwortung für die Entwicklung ihrer Heimatgemeinde und/oder für ihre Region. Voraussetzung dafür ist, dass sie sich in ihrer Region „gebunden“ fühlen und sich mit dieser (ländlichen) Region identifizieren. Somit ist es für Regionalplanung und -management, kommunale Planung und Politik wichtig, sich mit der frühzeitigen Einbindung und Mitgestaltung von Multilokalen zu befassen. Eine Möglichkeit besteht darin, das bürgerschaftliche Engagement von multilokal lebenden Personen als „Standort-, Bleibe- und Rückkehrfaktor" für den gesellschaftlichen Zusammenhalt in ländlichen Orten und Regionen zu fördern. Mögliche Aufgaben des Regionalmanagements könnten in der Schaffung geeigneter Rahmenbedingungen für die Etablierung und Umsetzung freiwilliger Tätigkeiten, der Errichtung geeigneter Vernetzungsstrukturen und -möglichkeiten sowie dem Zusammenbringen verschiedener Akteur*innen liegen. Dabei können zum Beispiel regionale Informationsplattformen entwickelt und zur Verfügung gestellt werden, die bei der Vermittlung von zivilgesellschaftlichem Engagement helfen (vgl. Beitrag Greinke/Lehmann/Othengrafen/Seitz in diesem Band (Kap. 7)).

\section{Arbeit und regionale Wirtschaft}

Im Handlungsfeld Arbeit und regionale Wirtschaft gilt es für das Regionalmanagement - ggf. zusammen mit der regionalen Wirtschaftsförderung - vornehmlich eine gezielte Zusammenarbeit zwischen Unternehmen und Kommunen zu fördern, um positive Bedingungen für multilokale (Fach-)Arbeitskräfte zu schaffen. Kommunen und Unternehmen sollten für multilokale (Fach-)Arbeitskräfte attraktiv sein und dies über die Schaffung spezieller Angebote realisieren: Dazu zählen zum Beispiel flexible Arbeitszeit-Modelle, Home-Office-Lösungen oder eine Unterstützung bei der Wohnungssuche und -vermittlung. Zudem können Kommunen mit Unterstützung des Regionalmanagements die regionale Außendarstellung verbessern, um sich insgesamt als Wirtschaftsregion zu 
präsentieren und im interregionalen Wettbewerb konkurrieren zu können. Dabei könnten sie auch ihre weichen Standortfaktoren herausstellen. Das Regionalmanagement kann gemeinsam mit Unternehmen. Regionalplanung, kommunaler Planung und Politik sowie Multilokalen und der lokalen Zivilgesellschaft Entwicklungskonzeptionen erarbeiten. Insbesondere Unternehmen werden immer internationaler, sodass die Standortanforderungen - auch von Multilokalen - steigen und somit gemeinsame Konzepte und Strategien erforderlich sind. Unterstützend kann dabei der Aufbau eines regionalen Netzwerkes / Regionalmanagements als Organisatorin des regionalen Austausches sein. Deutlich wird, dass die Regionalplanung in diesem Handlungsfeld eher wenig Handlungsmöglichkeiten hat, das Regionalmanagement aber durchaus unterstützend wirken kann.

\subsection{Multilokalität als Chance oder Risiko für die Regionalentwicklung?}

Multilokalität bedingt zahlreiche Chancen und Risiken für Kommunen (vgl. Beiträge Albrecht/Dittrich-Wesbuer (Kap. 3), Greinke/Lange (Kap. 4) sowie Greinke/ Albrecht/Othengrafen/Lehmann/Seitz in diesem Band (Kap. 5)). Ob und inwiefern multilokale Lebensweisen eine Chance oder Herausforderung für die Entwicklung ländlicher Räume sein können, hängt oft von den Motiven und Ausprägungen multilokaler Lebensweisen sowie der Entwicklung der Kommunen ab (vgl. Beiträge Albrecht/Dittrich-Wesbuer (Kap. 3), Greinke/Lange (Kap. 4) sowie Greinke/Albrecht/Othengrafen/Lehmann/Seitz in diesem Band (Kap. 5)). Ausschlaggebend sind verschiedene Multilokalisierungstreiber (s. o.), die begünstigen, dass Menschen phasenweise an- und abwesend sind (s.a. Greinke 2020). Zudem spielt die Lage und Ausstattung ländlicher Räume eine entscheidende Rolle. Um sich der Frage nach Chancen und Herausforderungen beispielhaft anzunähern, werden nachfolgend vier unterschiedlich charakterisierte Typen ländlicher Räume (vgl. Beitrag Geinke/Lange/Born in diesem Band (Kap. 2)) im Hinblick auf mehrörtige Lebensweisen reflektiert:

- Wirtschaftsstarke ländliche Räume: Diese Räume sind vorrangig geprägt durch Incomings, die aufgrund von oft großen, überregional tätigen Unternehmen ,,angezogen“ werden. Gleichzeitig besteht für die wirtschaftsstarken Räume zunehmend die Herausforderung (Fach-)Arbeitskräfte zu gewinnen und dauerhaft zu binden. Die Regionalentwicklung steht hier folglich vor der Chance, phasenweise Zuziehenden gute, attraktive Möglichkeiten zu bieten; gleichzeitig ergibt sich hier allerdings die Herausforderung, zeitweise Anwesende dauerhaft zu binden. 
- Periphere (strukturschwache) ländliche Räume: Gekennzeichnet durch eine Lage entfernt von Zentren und damit geringer infrastruktureller und funktionaler Verflechtung stehen diese Räume vor zahlreichen Herausforderungen, gleichwertige Lebensverhältnisse zu gewährleisten. Hier kann eher davon ausgegangen werden, dass es in peripheren Räumen weniger multilokale Incomings gibt. Allerdings ist mit mehrörtigen Outgoings zu rechnen, die aufgrund fehlender wohnortnaher Arbeitsplätze die Kommunen zeitweise. Für Regionalplanung und -management liegt der Fokus in diesen Räumen auf der Sicherstellung der Daseinsvorsorge und der Schaffung eines attraktiven Lebensumfelds und guter Versorgungsstrukturen, um Outgoings und deren Haushaltsmitglieder langfristig in der Region zu halten. Damit können unter Umständen das bürgerschaftliche Engagement und der gesellschaftliche Zusammenhalt gestärkt werden, auch wenn einzelne Arbeitnehmende temporär abwesend sind. Gleichzeitig können diese Räume aber auch von Multilokalität profitieren, indem sie beispielsweise Einnahmen durch Steuern und Gebühren (z. B. gemeindlicher Einkommenssteueranteil, kommunaler Finanzausgleich etc.) generieren, die die (kommunale) Daseinsvorsorge sicherstellen. Hier kommt es darauf an, dass Multilokalität nicht eine Vorstufe der endgültigen Abwanderung in dynamische Zentren mit attraktiven Arbeitsmärkten ist, sondern zur Stabilisierung der strukturschwachen Region durch „Rücktransfer“ von Einkommen und „Sozialkapital“ (Ideen, Kontakten usw.) beiträgt.

- Touristisch geprägte ländliche Räume: Freizeitinfrastrukturen sind in diesen Räumen meist gut entwickelt und ausgebaut. In diesen Räumen sind in großem Umfang Ferienhaussiedlungen, Dauercampingplätze oder andere Freizeitinfrastrukturen zu finden, die zu freizeitlich bedingten mehrörtigen Lebensweisen führen (vgl. Beitrag Othengrafen/Greinke/Lange/Seitz in diesem Band (Kap. 1)), auf die Regionalplanung und -management reagieren können. Das kann sowohl bei der Infrastrukturausstattung als auch in der Ausgestaltung von sozialen Angeboten geschehen. $\mathrm{Zu}$ vermuten ist, dass Kommunen in touristisch geprägten ländlichen Räumen bereits (oftmals unbewusst) mit Multilokalität umgehen: Dabei kann Mehrörtigkeit eine Chance sein, die Kommunen attraktiv zu gestalten, z. B. durch den Ausbau sozialer oder technischer Infrastrukturen oder die Vergrößerung des Einzelhandelsangebotes. Zugleich kann Multilokalität durch die temporären An- und Abwesenheiten zu Über- und Unterauslastungen bei den technischen Infrastrukturen (Wasser-/Abwassernetze etc.) führen, eingeschränkte Öffnungszeiten des Einzelhandels in weniger nachgefragten Jahreszeiten nach sich ziehen oder negative Auswirkungen auf das Erscheinungsbild von Kommunen (z. B. durch geschlossene Jalousien; Dirksmeier 2012) haben. Insgesamt 
können Regionalplanung und -management hier zwar entsprechende Rahmenbedingungen schaffen (z. B. Begrenzungen bei Flächenausweisungen etc.) und relevante Akteur*innen zusammenbringen; die detaillierten Planungen und Entscheidungen werden aber von den Kommunen getroffen.

- Agrarisch geprägte ländliche Räume: Räume mit zahlreichen landwirtschaftlichen Flächen und Betrieben sind oftmals durch phasenweise Anwesenheiten von Saisonarbeiter*innen und Erntehelfer*innen insbesondere in den Erntemonaten geprägt. Lediglich in den Wintermonaten sind eher selten Incomings anwesend. Für das Regionalmanagement bedeutet dies, mit zeitweisem Zuzug umzugehen, der sich auf das Sommerhalbjahr konzentriert. Damit hängt zusammen, dass beispielsweise Wohnraum phasenweise stark nachgefragt ist und gleichzeitig zeitweise leer steht. In der Verantwortung stehen hier insbesondere agrarische Betriebe, die die Bedürfnisse der Beschäftigten eruieren und entsprechend ausgestatteten Wohnraum sowie weitere Infrastrukturen für die Saisonarbeiter*innen und Erntehelfer*innen bereitstellen sollten. Das Regionalmanagement kann unterstützend agieren, in dem es Bedarfe sondiert sowie Akteur*innen zusammenbringt (z. B. Mehrfachnutzungen der Unterkünfte durch verschiedene Betriebe o.ä.) und für Multilokalität sensibilisiert. Die formelle Regionalplanung nimmt bei diesen Fragen keine aktive Rolle ein, weil sie zum Beispiel in der Wohnraumversorgung nur bedingt verantwortlich ist (s. o.).

Es wird deutlich, dass multilokale Lebensweisen vielfältig sind, dass sie in unterschiedlichen Raumtypen vielfältige Formen annehmen und dort vielschichtige Auswirkungen haben können, auf die Kommunen und andere Akteur*innen reagieren sollten. Regionalplanung und -management haben verschiedene Handlungsmöglichkeiten und Handlungsfelder (s. o.), die sie mehr oder weniger stark nutzen bzw. nutzbar machen können, um Chancen der Multilokalität für die Regionalentwicklung zu ergreifen und Risiken der Multilokalität für die Regionalentwicklung zu mindern.

\section{4 "Gretchenfrage": Multilokalität fördern oder Multilokale dauerhaft sesshaft machen?}

Ist Multilokalität ein Phänomen, auf das reagiert werden muss? Wenn ja, wer sind die Akteur*innen, die Handlungsansätze und Strategien im Umgang mit Multilokalität entwickeln müssen? Ist es sinnvoll, Multilokalität zu fördern oder Multilokale dauerhaft sesshaft zu machen? Im Projekt TempALand wurde deutlich, dass sich politisch-planerische, kommunale oder wirtschaftliche Akteur*innen i. d. R. bislang kaum mit Multilokalität beschäftigt haben. Aus diesem Grund ist 
es wichtig, die Akteur*innen für eine mehrörtige Lebensweise zu sensibilisieren. Dafür wurden zwei zentrale Strategien für den Umgang mit Multilokalität identifiziert: 1.) Attraktive Lebensbedingungen für Multilokale zu schaffen und 2.) Multilokale sesshaft zu machen. Dabei kann es sinnvoll sein, die beiden Strategien nicht als gegenseitig ausschließend, sondern verknüpfend und ergänzend zu betrachten (vgl. Beitrag Greinke/Lehmann/Othengrafen/Seitz in diesem Band (Kap. 7)).

Deutlich wird dabei, dass die „Gretchenfrage“, ob Multilokalität gefördert oder Multilokale dauerhaft sesshaft gemacht werden sollten, in jeder Kommune und Region differenziert betrachtet werden muss. In wirtschaftsstarken ländlichen Räumen ist es unter Umständen (politisch) gewollt, Multilokale von den Standortqualitäten der Region zu überzeugen und zu einem dauerhaften Umzug in die Region zu überzeugen. In eher strukturschwachen ländlichen Räumen wird Multilokalität evtl. eher ,geduldet“ oder unterstützt, da diese Räume - trotz temporärer Abwesenheiten einzelner Arbeitnehmer*innen - von Multilokalität profitieren, indem sie beispielsweise Einnahmen durch Steuern und Gebühren generieren, die die (kommunale) Daseinsvorsorge sicherstellen (s. o.). Regionalplanung und -management sollten Handlungsansätze und Strategien im Umgang mit Multilokalität je nach Ausgangslage und in Abhängigkeit von politischen Entscheidungen entwickeln und anpassen. Dabei können sich die formale Regionalplanung und das informelle Regionalmanagement gegenseitig unterstuitzen und beratende, vernetzende Aufgaben für die Kommunen, Unternehmen und Zivilgesellschaft übernehmen. Die Regionalplanung kann dabei insbesondere über das Zentrale-Orte-Konzept steuernd eingreifen, um der lokalen Bevölkerung und den Multilokalen Mindeststandards der Daseinsvorsorge zu ermöglichen. Zudem kann beispielsweise die Wohnraumversorgung und Ansiedlung von Gewerbe (als möglicher Arbeitgeber für Multilokale) durch gezielte und kontrollierte Flächenausweisungen gesteuert werden. Insgesamt ist die Steuerungswirkung der Regionalplanung in Bezug auf Multilokalität jedoch eher gering (s. o.). Das Regionalmanagement hingegen kann durch seine Vernetzungsstrategien Akteur*innen zusammenbringen und ggf. in Kooperation mit anderen Akteur*innen (z. B. der Wirtschaftsförderung, Vereinen und Verbänden etc.) regionale Kooperationen auf- und ausbauen. Dem Regionalmanagement kommt demnach eine stärkere Steuerungswirkung zu. Nichtsdestotrotz ist Multilokalität sehr von der individuellen Lebenssituation abhängig, die stets auch von individuellen Entscheidungen bezüglich der Ausgestaltung, Wohnortwahl, Beteiligung etc. bestimmt wird und demnach aus Sicht der Regionalplanung und des Regionalmanagements insgesamt schwer greifbar ist, sodass ein Umgang mit der Lebensweise und den daraus resultierenden Auswirkungen eine Herausforderung bleibt.

Deshalb sollten zukünftige Diskussionen um Gleichwertigkeit und die Abmilderung regionaler Disparitäten nicht losgelöst vom Phänomen Multilokali- 
tät erfolgen, denn multilokale Lebensweisen sind und werden für die räumliche Planung und die Sicherstellung gleichwertiger Lebensverhältnisse immer bedeutsamer (vgl. Beiträge Greinke/Albrecht/Othengrafen/Gutsche/Lehmann (Kap. 5) und Greinke/Lehmann/Othengrafen/Seitz in diesem Band (Kap. 7)). Multilokalität als fluide und hybride Lebensweise ist derart divers, dass sie sich auf nahezu alle Handlungsfelder der Regionalplanung und des Regionalmanagements auswirkt und deshalb zumindest immer mitgedacht werden sollte. Es gilt, Akteur*innen für das Phänomen zu sensibilisieren, um Chancen der mehrörtigen Lebensweisen zu erkennen und Herausforderungen aufzugreifen.

\section{Literatur}

Blatter, J. (2004): From 'spaces of place' to spaces of flow'? International Journal of Urban and Regional Research, 28, 530-548.

Blotevogel, H. (Hg.) (2002): Fortentwicklung des Zentrale Orte Konzepts. ARL Forschungs- und Sitzungsberichte, Hannover.

Bundesministerium des Innern, für Bau und Heimat (BMI) u.a. (2019): Unser Plan für Deutschland; Berlin.

Bundesministerium für Verkehr und digitale Infrastruktur (BMVI) (Hg.) (2015): Aktionsprogramm regionale Daseinsvorsorge. Projektassistenz Umsetzungsphase. BMVIOnline-Publikation 04/2015 (Bearbeiter: Dehne, P.; Kaether, J. und Neubauer, A.), URN: https://www.bbsr.bund.de/BBSR/DE/veroeffentlichungen/ministerien/bmvi/ bmvi-online/2015/DL_BMVI_Online_04_15.pdf?_blob=publicationFile \&v=1.

Danielzyk, R./Dittrich-Wesbuer, A. (2020): Multilokalität in der Stadt- und Regionalentwicklung. In: Danielzyk, R./Dittrich-Wesbuer, A./Hilti, N./Tippel, C. (Hg.) (2020): Multilokale Lebensführungen und räumliche Entwicklungen - ein Kompendium. Hannover. Forschungsberichte der ARL 13. URN : https://nbn-resolving.de/ urn:nbn:de:0156-09764, 196-202.

Danielzyk, R./Priebs, A. (2020): Die Sicherung der Daseinsvorsorge durch Klein- und Mittelstädte als Beitrag des zentralörtlichen Systems zu gleichwertigen Lebensverhältnissen. Europa Regional, 3-2018(2020), 7-21.

Einig, K./Grabher, G./Ibert, O./Strubelt, W. (2005): Urban Govrnance - Einführung, Informationen zur Raumentwicklung, Heft 9/10.2005, I-IX.

Fachinger, U./Künemund, H. (Hg.) (2015): Gerontologie und ländlicher Raum. Lebensbedingungen, Veränderungsprozesse und Gestaltungsmöglichkeiten. Vechtaer Beiträge zur Gerontologie. Wiesbaden: Springer VS Verlag.

Fischer, T. (2020): Soziale Infrastrukturen und Multilokalität. In: Danielzyk, R./DittrichWesbuer, A./Hilti, N./Tippel, C. (Hg.) (2020): Multilokale Lebensführungen und räumliche Entwicklungen - ein Kompendium. Hannover. = Forschungsberichte der ARL 13. URN : https://nbn-resolving.de/urn:nbn:de:0156-09764, 140 - 146.

Fürst, D./Lahner, M./Pollermann, K. (2006): Entstehung und Funktionsweise von Regional Governance bei dem Gemeinschaftsgut Natur und Landschaft. Analysen von Placemaking- und Governance-Prozessen in Biosphärenreservaten in Deutschland und 
Großbritannien. Beiträge zur räumlichen Planung. Schriftenreihe der Fachgruppe Landschaft, Fakultät für Architektur und Landschaft der Leibniz Universität Hannover, Heft 82, Hannover.

Greinke, L. (2020): Berufsbedingte Multilokalität in ländlichen Räumen Niedersachsens. Gesellschaftliche und räumliche Auswirkungen als planerische Herausforderung am Beispiel des Landkreises Diepholz. Ländliche Räume: Beiträge zur lokalen und regionalen Entwicklung Bd. 7, LIT-Verlag Berlin.

Heintel, M. (2018): Regionalmanagement. In: ARL-Akademie für Raumforschung und Landesplanung (Hrsg.): Handwörterbuch der Stadt- und Raumentwicklung. Hannover, URN: https://nbn-resolving.de/urn:nbn:de:0156-55993, 2023 - 2028.

Hilti, N. (2009): Multilokales Wohnen: Bewegungen und Verortungen. Informationen zur Raumentwicklung ,1/2.2009, 77-86.

Jann, W. (2002): Der Wandel verwaltungspolitischer Leitbilder: Vom Management zu Governance? In: König, K. (Hg.): Deutsche Verwaltung an der Wende zum 21. Jahrhundert, Baden-Baden, 279-303.

Kersten, J. (2006): Abschied von der Gleichwertigkeit der Lebensverhältnisse - der „wirtschaftliche, soziale und territoriale Zusammenhalt "als neue Leitvorstellung der Raumordnung, Umwelt-und Planungsrecht 2006/7, 245-252.

Knieling, J. (2018): Raumentwicklung. In: ARL-Akademie für Raumforschung und Landesplanung (Hrsg.): Handwörterbuch der Stadt- und Raumentwicklung. Hannover, URN: https://nbn-resolving.de/urn:nbn:de:0156-55993, 1873 - 1880.

Lange, L. (2018): Leben an mehreren Orten. Multilokalität und bürgerschaftliches Engagement in ländlich geprägten Räumen Niedersachsens. Berlin: LIT Verlag.

Oberösterreichische Zukunftsakademie (Hg.) (2019): Leben an mehreren Orten. Multilokalität als Chance für Stadt und Land. Linz.

Peer, V. (2013): Dezentrale tertiäre Ausbildungsstätten und ihr Einfluss auf das Abwanderungs- und Bleibeverhalten Hochqualifizierter in ländlichen Regionen Österreichs. Eine raumwissenschaftliche Analyse am Beispiel ausgewählter FachhochschulStandorte. Dissertation am Institut für Raumplanung und Ländliche Neuordnung, Universität für Bodenkultur. Wien.

Priebs, A. (2018): Regionalplanung. In: ARL-Akademie für Raumforschung und Landesplanung (Hrsg.): Handwörterbuch der Stadt- und Raumentwicklung. Hannover, URN: https://nbn-resolving.de/urn:nbn:de:0156-55993, 2047 - 2062.

Rosenfeld, M. T. W. (2018): Gleichwertigkeit der Lebensverhältnisse. In: ARL - Akademie für Raumforschung und Landesplanung (Hrsg.): Handwörterbuch der Stadt- und Raumentwicklung. Hannover, $837-849$.

Scharmann, L./Priebs, A./Einig, K./Janssen, G./Stefansky, A. (2020): Raumordnung: Anwalt für gleichwertige Lebensverhältnisse und regionale Entwicklung. Eine Positionsbestimmung, Positionspapier aus der ARL, No. 115, Verlag der ARL Akademie für Raumentwicklung in der Leibniz-Gemeinschaft, Hannover, https://nbnresolving.de/urn:nbn:de:0156-0115.

Terfrüchte, T./Flex, F. (2018): Zentraler Ort. In: ARL-Akademie für Raumforschung und Landesplanung (Hrsg.): Handwörterbuch der Stadt- und Raumentwicklung. Hannover, URN: https://nbn-resolving.de/urn:nbn:de:0156-55993, 2969 - 2979. 
ARL - Akademie für Raumforschung und Landesplanung (Hg.) (2016): Multilokale Lebensführung und räumliche Entwicklungen. Positionspapier aus der ARL 104. Hannover.

Dirksmeier, P. (2012): Multilokalität als Abwesenheit: eine Herausforderung für landschaftlich attraktive ländliche Räume - das Beispiel Tegernsee/Stadt. Europa Regional 18, 2-3, 60-70.

Leubert, N. (2020): Annäherung an die berufsbedingt Multilokalen in Wolfsburg. In: Danielzyk, R./Dittrich-Wesbuer, A./Hilti, N./Tippel, C. (Hg.) (2020): Multilokale Lebensführungen und räumliche Entwicklungen - ein Kompendium. Hannover. Forschungsberichte der ARL 13. URN : https://nbn-resolving.de/ urn:nbn:de:0156-09764, 243-252.

Wiegandt, C.-C./Krajewski, C. (2020): Einblicke in ländliche Räume in Deutschland - zwischen Prosperität und Peripherisierung. In:Krajewski, C./ Wiegandt, C.-C. (Hg.) (2020): Land in Sicht. Ländliche Räume in Deutschland zwischen Prosperität und Peripherisierung. Bundeszentrale für politische Bildung, 12-32.

Open Access Dieses Kapitel wird unter der Creative Commons Namensnennung 4.0 International Lizenz (http://creativecommons.org/licenses/by/4.0/deed.de) veröffentlicht, welche die Nutzung, Vervielfältigung, Bearbeitung, Verbreitung und Wiedergabe in jeglichem Medium und Format erlaubt, sofern Sie den/die ursprünglichen Autor(en) und die Quelle ordnungsgemäß nennen, einen Link zur Creative Commons Lizenz beifügen und angeben, ob Änderungen vorgenommen wurden.

Die in diesem Kapitel enthaltenen Bilder und sonstiges Drittmaterial unterliegen ebenfalls der genannten Creative Commons Lizenz, sofern sich aus der Abbildungslegende nichts anderes ergibt. Sofern das betreffende Material nicht unter der genannten Creative Commons Lizenz steht und die betreffende Handlung nicht nach gesetzlichen Vorschriften erlaubt ist, ist für die oben aufgeführten Weiterverwendungen des Materials die Einwilligung des jeweiligen Rechteinhabers einzuholen.

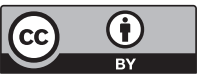

